\title{
On Some Generalization of the Rayleigh Problem on a Convective Instability
}

\author{
Lev Kh. Ingel \\ Research and Production Association 'Typhoon', Pobedy 4, Obninsk, Kaluga Region, 249038, Russia \\ *Corresponding Author: lev.ingel@gmail.com
}

Copyright (C) 2014 Horizon Research Publishing All rights reserved

\begin{abstract}
Previous investigations using the numerical modeling of moist convection in the atmosphere found an interesting effect: clouds arising through the water vapor condensation shield partially the underlying surface and change its radiation balance. The vertical heat fluxes on the surface become horizontally inhomogeneous, that can exert a back profound effect on the convection and dynamics of clouds, in particular, resulting in their horizontal transference (a cloud "runs away from its own shadow"). This paper is dedicated to the corresponding generalization of the classical Rayleigh problem on the convective instability of horizontal layer of a fluid. This generalization has as its object to describe the effect mentioned above - the influence of partial surface shielding on convection. The results show that the given relatively small modification of the Rayleigh problem, taking into account the possibility of the horizontally shifted thermal response to the vertical motions, leads to qualitatively new results. There appears a new, easily realizable type of instability, for which the strengthening of disturbances moving horizontally is typical.
\end{abstract}

Keywords Convective instability, Rayleigh problem, Atmosphere, Clouds

\section{Introduction}

This note is dedicated to some generalization of the classical Rayleigh problem on the convective instability of horizontal layer of a fluid. This generalization is related to certain peculiarities of the convection in the low atmosphere in the presence of clouds. Clouds can partially screen the sun irradiation that results in horizontally inhomogeneous heating of the layer from the bottom.

In some studies dedicated to analysis of the moist cellular convection in the atmospheric boundary layer over the land, it was found that cooling of the surface in the shadow of arising clouds can affect the structure of convective cells and the convective regimes $[1-3]$. The vertical heat flux from the surface becomes horizontally inhomogeneous, that affects the dynamics of convection and convective clouds, and in particular, results in the horizontal movement of cells (a cloud "runs away from its own shadow").

One can assume that a range of meteorologically significant phenomena, arising under inhomogeneous screening the underlying surface by clouds, may be rather wide. The formation of horizontal thermal inhomogeneities should also affect the threshold of convective stability. Let us consider a case when a disturbance in the form of ascending motions arises in an area over the underlying surface; these motions have strengthened a condensation and resulted in the additional heat releasing and may lead to formation of clouds that reduce of the air clarity. If the Sun is angularly to the zenith, then screening of horizontally neighboring surface area may strengthen. A horizontal contrast of temperatures arises (or strengthens) that in some situations can promote the growth of the initial disturbance (of the ascending motions), besides the above mentioned the horizontal motion of disturbances. The emergent horizontal contrast of temperatures (or of vertical heat fluxes) can be significant, taking into account the small effective heat capacity of several widespread types of the underlying surface found lately $[4,5]$.

In such situations, there is a principal physical distinction from conventional problems on convection. In the conventional problems, the vertical motions result in the formation (appearance) of temperature disturbances along vertical updrafts or downdrafts. But in the case under consideration, a thermal response may be partially shifted horizontally; a thermal response takes place not only in the area of ascending motions, where a cloud arises, but also along another vertical, where a cloud shadow drops. Such "shifting of thermal response" results in a qualitatively new behavior of disturbances.

In particular, it is interesting to generalize the Rayleigh problem on convective instability by taking into account the forementioned additional feedbacks. The present note considers a simplified analytical model, which allows one to start the systematic analysis of such phenomena.

\section{Modification of the Rayleigh Problem by Implementation of Additional Horizontally Shifted Heat Response}


Into the system of equations of hydrothermodynamics, an additional horizontally inhomogeneous source (sink) of heat $Q$ is introduced to describe the effects of screening of insolation. For simplicity, let us restrict our consideration by two-dimensional problem. The linearized system of equations of dynamics, continuity and heat transfer in the Boussinesq approximation is:

$$
\begin{gathered}
\frac{\partial u}{\partial t}=-\frac{\partial \Phi}{\partial x}+v \Delta u, \quad \frac{\partial u}{\partial x}+\frac{\partial w}{\partial z}=0, \quad \Delta=\frac{\partial^{2}}{\partial x^{2}}+\frac{\partial^{2}}{\partial z^{2}}, \\
\frac{\partial w}{\partial t}=-\frac{\partial \Phi}{\partial z}+v \Delta w+\alpha g \theta, \quad \frac{\partial \theta}{\partial t}+\Gamma w=\kappa \Delta \theta+Q .
\end{gathered}
$$

Here $u, w$ are the components of velocity along horizontal and vertical axes $x$ and $z$, respectively; $t$ is the time, $\theta$ is a potential temperature disturbance, $\Phi$ is a pressure disturbance referred to the average density of medium (air); $\Gamma$ is a background vertical gradient of potential temperature (this is positive at the stable stratification); $v, \kappa$ are the exchange coefficients for the proper substances; $\alpha$ is the thermal coefficient of the air expansion, $g$ is the gravitational acceleration.

A horizontal layer $0 \leq z \leq H$ is under consideration. On the horizontal boundaries there is supposed a fulfillment of the Rayleigh boundary conditions, in particular, the condition

$$
w=0 \text { at } z=0, H .
$$

Slip boundary conditions are applied.

The solution for the first vertical mode is sought in the form of normal waves

$$
w \sim \sin \lambda z \cdot \exp [i(k x-\omega t)], \quad \lambda=2 \pi / H .
$$

Here $k$ is the wave number, $\omega$ is the complex increment of disturbances. Let us consider an explicit form of the source (sink) of heat $Q$, parametrizing the effects of screening the surface by clouds. Obviously, this source has to depend on the velocity of vertical air motions, which determine the existence and intensity of the convective clouds. But, except for the case when the Sun is in the zenith, the screening effect of cloud is shifted relative to this cloud horizontally. Therefore, the most important circumstance for the mechanism under consideration consists in the fact that the considered additional sink of heat depends on cloud presence (in particular, on the presence of vertical motions) in some of neighboring horizontally areas. As the simplest model we assume that the sink of heat in the air column with horizontal coordinate $x$ is proportional to the vertical velocity in another air column, with some coordinate $x-d$, from which a shadow drops:

$$
Q(x, z, t)=-G w(x-d, z, t) .
$$

Here $d$ is the distance, which is assumed to be known from geometry of the problem (this primarily depends on the Sun height over the horizon), $G(\mathrm{~K} / \mathrm{m})$ is the positive coefficient of proportionality defining the effectiveness of screening.

Of course, such a model is greatly simplified. Indeed, the screening primarily affects the heat balance of underlying surface, and only through this - in the air column as a whole. But such simplest modification of the convective stability problem already results in substantial and physically clear results. Therefore, it seems to be reasonable to start with this modification.

\section{Solution}

Besides Rayleigh and Prandtl numbers and aspect ratio $a=k / \lambda$, there are some more two dimensionless parameters in the given problem, characterizing intensity $\left(\delta=G / \Gamma=\left(N_{G} / N\right)^{2}, \quad N^{2}=\alpha g \Gamma, \quad N_{G}^{2}=\alpha g G>0\right)$ and horizontal shift $(k d)$ of the feedback under consideration. Dispersive equation has a form

$$
\omega^{2}+i \omega\left(1+a^{2}\right) \lambda^{2}(v+\kappa)-\left(1+a^{2}\right)^{2} \lambda^{4} v \kappa-\frac{a^{2}}{1+a^{2}}\left(N^{2}+N_{G}^{2} e^{-i k d}\right)=0
$$

Denoting $\mathrm{Ra}_{*}=\left(1+a^{2}\right)^{3} / a^{2}$ - the expression indicated the neutral condition in the classical Rayleigh problem and $\mathrm{Ra}=-N^{2} / v \kappa \lambda^{4} \quad$ used in the conventional problem on convective stability with Rayleigh boundary conditions, Eq (1) can be written as

$$
\omega=\frac{1}{2}\left(1+a^{2}\right) \lambda^{2}(v+\kappa)\left\{-i \pm \sqrt{-1+\frac{4 v \kappa}{(v+\kappa)^{2}}\left[1-\frac{\mathrm{Ra}}{\mathrm{Ra}_{*}}(1+\delta \cos k d-i \delta \sin k d)\right]}\right\}
$$

At $K=V$ the latter expression is simplified noticeably: 


$$
\begin{aligned}
& \omega=\left(1+a^{2}\right) \lambda^{2} v\left\{-i \pm \sqrt{-\frac{\mathrm{Ra}}{\mathrm{Ra}_{*}}(1+\delta \cos k d-i \delta \sin k d)}\right\}, \\
& \operatorname{Im} \omega=\left(1+a^{2}\right) \lambda^{2} v\left\{-1 \pm \sqrt{\frac{|\mathrm{Ra}|}{2 \mathrm{Ra}_{*}}}\left[\left(1+2 \delta \cos k d+\delta^{2}\right)^{1 / 2}+\operatorname{sgn} \mathrm{Ra}(1+\delta \cos k d)\right]^{1 / 2}\right\} \\
& \operatorname{Re} \omega=\mp\left(1+a^{2}\right) \lambda^{2} v \sqrt{\frac{|\mathrm{Ra}|}{2 \mathrm{Ra}_{*}}}\left[\left(1+2 \delta \cos k d+\delta^{2}\right)^{1 / 2}-\operatorname{sgn} \operatorname{Ra}(1+\delta \cos k d)\right]^{1 / 2} .
\end{aligned}
$$

\section{Analysis of solution}

In the simplest case, when a background stratification and dissipative factors are lacking (at zero values of parameters $N, \kappa, v)$, from (1) it follows

$$
\omega= \pm \frac{a}{\sqrt{1+a^{2}}} N_{G}\left(\cos \frac{k d}{2}-i \sin \frac{k d}{2}\right)
$$

It is seen that positive increments $\operatorname{Im} \omega=\frac{a}{\sqrt{1+a^{2}}} N_{G} \sin \frac{k d}{2}$ always exist, with the exception of discrete multitude of values $k d=2 \pi n$, where $n=0, \pm 1, \pm 2 \ldots$. At the mentioned values of parameter $k d$, shadow of each cloud is situated exactly under the cloud (or under one of the near clouds). It means the negative feedback, i.e. an additional cooling from below in the area of ascending motions; this hinders strengthening of disturbances. The disturbances always move horizontally always, with the exception of the cases when $k d=(2 n+1) \pi$, when horizontal thermal gradients on the both sides from the cloud are symmetric, and movement is impossible due to considerations of symmetry. The maximal increments of disturbances correspond to the values $k d$, under which there is no horizontal motion of disturbances.

In another limiting case, with small values of coefficients of exchange $\kappa, v$, but in the presence of background stratification (vertical temperature gradient), i.e. at large absolute values of Rayleigh number, from (1) it follows

$$
\begin{gathered}
\omega= \pm \frac{a}{\sqrt{1+a^{2}}}\left[N^{2}+N_{G}^{2} \exp (-i k d)\right]^{1 / 2}, \\
\operatorname{Re} \omega= \pm \frac{a}{\sqrt{2\left(1+a^{2}\right)}}\left[\left(N^{4}+N_{G}^{4}+2 N^{2} N_{G}^{2} \cos k d\right)^{1 / 2}+N^{2}+N_{G}^{2} \cos k d\right]^{1 / 2}, \\
\operatorname{Im} \omega=\mp \frac{a}{\sqrt{2\left(1+a^{2}\right)}}\left[\left(N^{4}+N_{G}^{4}+2 N^{2} N_{G}^{2} \cos k d\right)^{1 / 2}-N^{2}-N_{G}^{2} \cos k d\right]^{1 / 2} .
\end{gathered}
$$

In general, the analysis of problem on stability shows that in the system under consideration there exists a new, easily realizing type of instability, whose characteristic feature is the strengthening of disturbances, moving horizontally. This is shown in Figures 1, 2. 


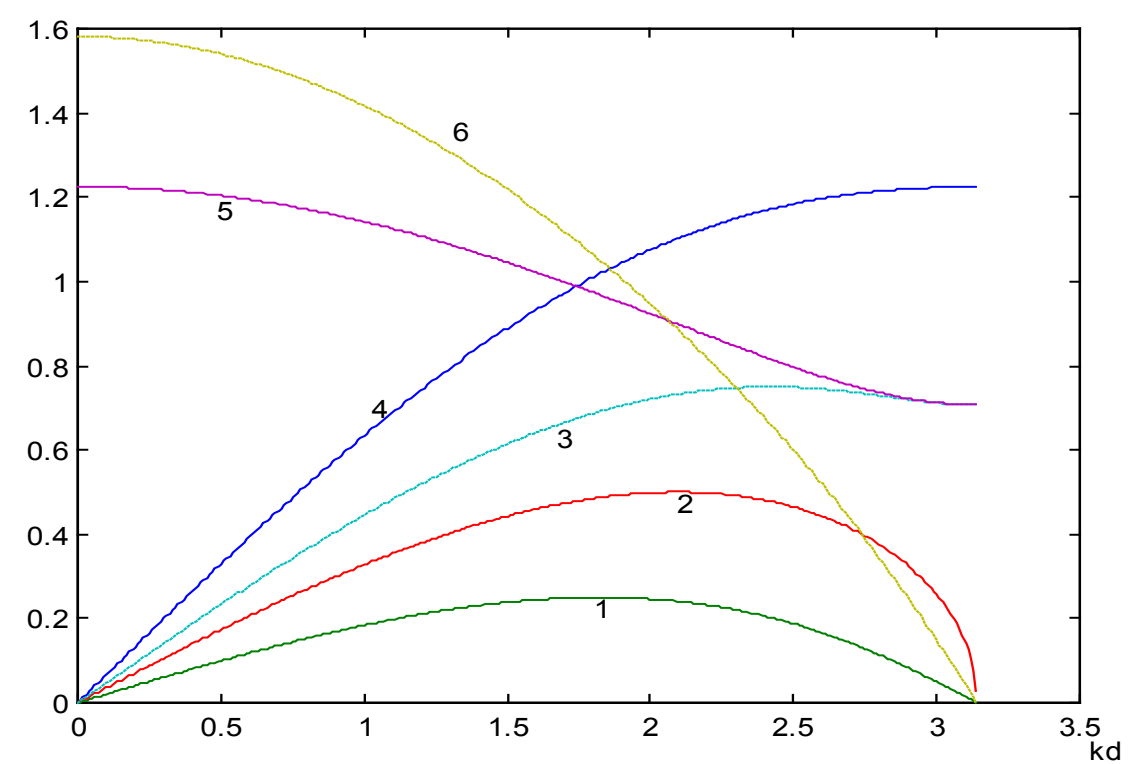

Figure 1. The dimensionless increments and velocities of disturbances moving horizontally at the stable background stratification and negligible values of coefficients of exchange (in the limit of large absolute values of Rayleigh number). Along the ordinate axis the imaginary part (curves 1-4) and taken with the negative sign the real part of $\omega$ (curves 5, 6), normalized on ${ }_{a N} /\left(1+a^{2}\right)^{1 / 2}$ are shown. The values of feedback amplitude are: $\delta=0.5 \quad(1)$,

$1(2), 1.5(3,6), 2.5(4,5)$.

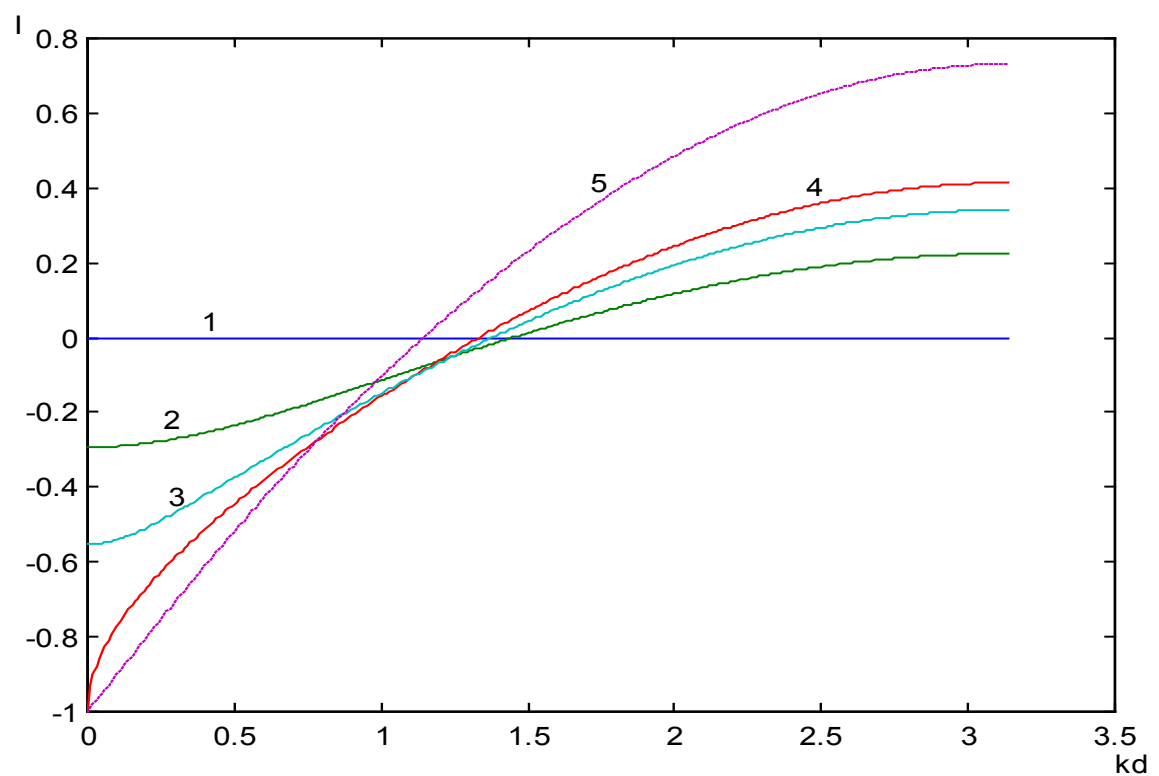

Figure 2. The dimensionless increments of disturbances $I=\operatorname{Im} \omega /\left(1+a^{2}\right) \lambda^{2} v$ depending on the dimensionless horizontal shift $k d$ at $\mathrm{Ra} / \mathrm{Ra}{ }_{*}=1$ for various values of the feedback amplitude: $\delta=0(1),-0.5(2),-0.8(3),-1(4),-2$ (5).

\section{Conclusion}

Thus, a rather small modification of the Rayleigh problem on the convective instability, taking into account the possibility of the horizontally shifted thermal response to the vertical motions, leads to qualitatively new results. There appears a new, easily realizable type of instability for which the strengthening of disturbances moving horizontally is typical. In respect to the dynamics of clouds, the considered model is, certainly, extremely simplified. We believe, however, that this model is a necessary step for the description of previously unstudied feedbacks in the lower troposphere.

\section{REFERENCES}

[1] A. P. Khain. Some Problems on Dry and Moist Convection in The Turbulent Atmosphere, Ph.D. dissertation, Institute of Experimental Meteorology, Obninsk, 1974 (in Russian). 
[2] V. N. Ivanov and A. P. Khain. The Role of Ground Screening by Cloudiness During Cellular Convection, Akademiia Nauk SSSR, Izvestiia, Fizika Atmosfery i Oceana, Vol. 11, No 10, 1975, 1063-1066 (in Russian; there is English translation: "Role of Screening of the Underlying Surface by Clouds in Cellular Convection", Izvestiya, Atmospheric and Oceanic Physics, Vol. 11, No 10, 1975, 666-668).

[3] N. L. Byzova, V. N. Ivanov and E. K. Garger. Turbulence in the Boundary Layer of the Atmosphere, Gidrometeoizdat, Leningrad, 1989 (in Russian).

[4] V. P. Kukharets, H. G. Nalbandyan, Th. Foken. Thermal Interactions Between the Underlying Surface and a Nonstationary Radiation Flux, Izvestiya, Atmospheric and Oceanic Physics, Vol. 36, No 3, 2000, 318-325 (in Russian).

[5] Th. Foken. Micrometeorology, Springer-Verlag, Berlin-Heidelberg, 2008 